III. Aus der chirurgischen Abtheilung des Vereins-Krankenhauses in Magdeburg.

\title{
Ein Vorschlag zur Behandlung der Fistula recti und des periproctitischen Abscesses.
}

Von Dr. Paul Sendler, dirig. Arzt.

Vielfache Beobachtnngen sprechen dafür, dass in unserer modernen Zeit die Erkrankungen des Darmcanals nnd damit anch seines nntersten Theiles, des Rectums, in fortwährend steigender Zunahme begriffen sind. Amerikanische Aerzte haben die Ansicht ausgesprochen, dass hierfür die Verhältnisse unserer heutigen iiberhasteten, nach raschem Erwerb jagenden Lebensweise verantwortlich zu machen seien, infolge deren die Menschen zu wenig auf Störuıgen ihrer Darmthätigkeit zn achten pflegten. $O b$ das richtig ist, und ob ein solcher Vorwurf auch für unsere deutschen Verhältnisse zutrifft, mag dahingestellt bleiben; für eine Anzahl von Fällen der Grossstadt lässt sich eine gewisse Wahrscheinlichkeit sicherlich nicht von der Hand weisen. Jedenfalls habe ich ans dem mir zufliessenden Krankenmaterial den Eindruck gewonnen, dass die Erkrankuugen an periproctitischen Abscessen und Fistula recti gegen frïhere Zeiten in allen Classen der Bevölkerung eine Steigerung erfahren haben.

Dass diese Leiden möglichst frühzeitig operativ in Angriff genommen werden mïssen, ist heute allgemein zngegeben, denn allein durch eine Operation ist hier die Möglichkeit der Heilung gewährleistet. Die mit einer solchen in frïheren Zeiten verbundenen Gefahren sind heute zum grössten Theil als überwnnden zn betrachten; unter normalen Verhältnissen und bei genügender Vorsicht kommt es wohl kanm noch vor, dass ein Kranker der Pyämie erliegt.

Während also die Prophylaxe bei dieser Operation eine geniigend sichere geworden ist, lässt sich nicht behaupten, dass die Technik derselben erhebliche Fortschritte gemacht habe. Zwar ist die veraltete Methode der Ligatur von Mastdarmfisteln als in unsere Anschauungen nicht mehr hineinpassend wohl allseitig verlassen worden, aber auch die Resultate der allgemein gebräuchlichen Operationsmethode, der Spaltung der Fistel mit Durchtrennung des M. sphincter ani externıs, können im allgemeinen wenig befriedigen.

Dass man zur Vermeidung des Blutverlustes und zum Schutze gegen die Infectionsgefahr hierbei zu unblutigen Methoden gegriffen hat, kanı kaum als Fortschritt bezeichnet werden. Denn mag man die Durchschneidung mit dem Messer, der galvanokaustisehen Schlinge oder dem Thermokauter ausfiihren: die Heilung der gesetzten Operationswunde muss durch Granulation erfolgen. Dieser Umstand bedingt erstens eine verhältnissmässig lange Heilungsdauer, die zudem durch das häufige nnvermeidliche Touchiren mit dem Höllensteinstift, aber auch sonst bei dem oft nöthigen Verbandwechsel fiir die Kranken mit vielfachen Schmerzen verbunden ist. A ber die Heilung durch Granulation hat noch eine andere sehr lästige Unbequemlichkeit im Gefolge. Während der ganzen langen Heilnugsdauer besteht Incontinentia alvi; dieselbe hält in den meisten Fällen auch nach vollendeter Heilung noch längere Zeit an und verliert sich für flüssigen Stuhl und Flatus in vielen Fällen erst sehr spät, iı manchen besonders schweren vielleicht auch gar nicht.

Alles das musste dazu drängen, nach einem Operationsverfahren zu suchen, durch welches solche Uebelstände vermieden werden. Schon seit längerer Zeit habe ich deshalb in dieser Richtıng Versuche angestellt. Dass zu diesem $Z$ weck die galvano- und thermokaustischen Methoden nicht zu gebrauchen waren, war voll vortlherein klar, nur von einer die Naht gestattenden blutigen Operation war die Möglichkeit eimer raschen und sicheren Heilung zu erwarten. Ich ging dabei von der Erwägung ans, dass es möglich sein mïsste, nach einer sorgfältig geleiteten Vorbereitungscur Verbältnisse zu 
schaffen, die gestatteten, das durchschnittene Darmrohr wieder zu schliessen und auch die Fistel, sowie die begleitenden periproctitischen Abscesse derartig herzurichten, dass auch sie durch Etagennaht einer vollen oder theilweisen prima reunio zugänglich gemacht würden. Die Ueberzeugung, dass dies möglich sein müsse, gewann ich bei einer im Anfang des Jahres 1886 ausgeführten Operation an einem Kinde, dem ich die durchschnittene Fistel und einen grossen periproctitischen Abscess anfrischte und nähte. Bei dem sehr unruhigen Knaben erreichte ich zwar keinen vollen Erfolg, aber doch eine wesentlich abgekürzte Heilungsdauer. Diese Erfahrung, an die sich noch andere ähnliche schlossen, sowie ein von Lange (NewYork) auf dem 17. Congress der Deutschen Gesellschaft für Chirurgie im Jahre 1887 in kurzen Worten gemachter Vorschlag (der nicht allgemein beachtet zu sein scheint und auch nicht in das Protokoll aufgenommen ist), die Fistel zu excidiren und zu nähen, bewogen mich, auf dem eingeschlagenen Wege fortzuschreiten.

Nach mancherlei Misserfolgen hat mir nun das nachfolgend beschriebene Verfahren in 11 hinter einander folgenden Fällen die besten Dienste geleistet.

Zunächst wird der Kranke, wie auch früher geschehen, einer zwei- bis dreitägigen Vorbereitungscur unterworfen. Er badet, bekommt Abführmittel und Darmausspülungen, bis man die Gewissheit hat, dass kein Darminhalt mehr das Operationsfeld verunreinigen wird. Kurz vor der Operation wird die Umgebung des Afters rasirt, abgeseift, mit in Aether getränkter Watte sorgfältig abgerieben und mit $1 \%$ Sublimatlösung energisch abgewaschen. Hierauf wird das Rectum selbst noch einmal mit $3 \%$ Borsäurelösung ausgespült.

Der tief narkotisirte Kranke befindet sich in Rückenlage mit erlıötem Becken, die Oberschenkel werden scharf im Hüftgelenk flectirt gehalten. Nun wird zunächst in gewöhnlicher Weise eine Hohlsonde von der äusseren Fistelöffnung her in das Rectum geführt und zur Analöffnung herausgeleitet, und auf dieser Sonde die Fistel sammt Schleimhaut und Sphincter externus gespalten. Nach Stillung der meist unerheblichen Blutung durch Compression wird jetzt mit Pincette und Scheere oder Messer die ganze Fistel exstirpirt, sodass überall blutendes gesundes Gewebe zu 'Tage liegt. In derselben Weise werden Recessus und etwaige Abscesse breit gespalten, die Granulationen herausgeschabt, die Abscesswände abgetragen, bindegewebige Brücken oder Scheidewände herausgeschnitten, sodass auch hier überall normales Gewebe zum Vorschein kommt und der Abscess womöglich in eine einzige glattwandige Höhle verwandelt wird. Die Blutung ist hierbei verhältnissmässig gering und lässt sich jedenfalls beherrschen. Meist genügt eine kurze Compression, spritzende Arterien werden unterbunden.

Der Hauptact der Operation ist die jetzt folgende genaue Vereinigung der Wundflächen durch die Naht. Bei grossen Abscessen ist eine sorgfältige, von der Tiefe her beginnende Etagennaht unerlässlich, bei kleineren lässt sich die Vereinigung sehr gut in der bei Lawson Taits Methode der Dammplastik gebrüuchlichen Weise der Naht bewerkstelligen, aber auch bei grossen Abscessen führe ich die tiefsten Nähte unter dem Grunde der Wunde hindurch. - Zuletzt wird die Fistel ganz iı derselben Weise genäht, wobei darauf zu achten ist, dass auch die Ränder der dnrchschnittenen Muskulatur wieder genau vereinigt werden. Den Schluss bildet die Naht der Schleimhaut des Rectums. Hat die Spaltung hoch hinauf stattgefunden, so wird bei dieser Vornahme am besten ein Rinnenspeculum eingesetzt.

Als Nähmaterial empfiehlt sich für die versenkten und Schleimhautnähte sorgfältig zubereitetes und desinficirtes Catgut verschiedener Stärke, das vollkommen ausreicht; nur für die Hautränder pflege ich ausschliesslich Jodoformseide zu verwenden.

In dieser Weise gestaltet sich das Verfahren in den einfacherell Fällen, bei welchen man höchstens mit Abscessen auf einer Seite des Rectums zu thun hat. Aber auch bei den schweren, ausgedehnten Abscessbildungen, bei denen wir den grössten Theil des Mastdarms abgehoben und umspült finden, lässt sich wenigstens cin Theil des Operationsterrains jedesmal in der beschriebenen Weise herrichten. Den Rest kann man zuweilen durch Hülfsschnitte noch der Naht zugänglich machen. Geht auch das nicht an, so wird man durch eine energische Ausschabung die Abscesswände säubern und nach dem tiefsten Punkte drainiren. In solchen Fällen kann sich die Vornahme zu einer plastischen Operation gestalten, deren zweckmässigste Anlage der Invention des Einzelnen überlassen werden muss.

Der Verband der so versorgten Operationswunde ist höchst einfach. Nach Bepuderung der Nahtlinie der Schleimhaut und der äusseren Haut mit Jodoform empfieblt es sich bei grossen Fisteln und Abscessen, einen Jodoformgazetampon in das Rectum einzuführen, in leichteren Fällen ist diese Vorsicht entbehrlich. Im übrigen genügen etwas gekrïllte Jodoformgaze und Watte oder ein untergelegtes Mooskissen, das mittels einer doppelten 'T-Binde festgehalten wird, vollkommen allen Anforderungen.
Auch die Nachbehandlung ist die erdenkbar einfachste und schonendste. Der Kranke wird mit lose zusammengebundenen Beinen zu Bette gebracht und hat ungefähr fünf bis sechs Tage hindurch eine möglichst ruhige Lage einzuhalten. Während dieser ganzen Zeit erhält er Opium zur Anhaltung des Stuhls und ganz leichte Diät. Nach dieser Zeit werden die Hautnähte und, wo ihre Anwendung nöthig erschien, auch die Drains entfernt, und durch Ol. Ricini und Eingiessungen für leichte Stuhlentleerung gesorgt. Auch von ungeduldigen Kranken wird dieser Zwang gern ertragen, da sie keine Schmerzen oder sonstige Unbequemlichkeiten zu erleiden haben, sich im Gegentheil meist vollkommen schmerzfrei und behaglich fühlen.

Ist glatte prima intentio eingetreten, so ist hiermit die ganzo Behandlung beendigt, und thatsächlich sind einige meiner Kranken mit 5 und 6 Tagen vollständig geheilt gewesen. Ein so günstiges Resultat kann man aber natürlich nur in ganz leichten uncomplicirten Fällen erwarten. Meist ist noch eine nach allgemein gültigen chirurgischen Regeln durchzuführende Nachbehandlung nöthig, aber auch in den schwersten Fällen ist nach annähernd 4 bis 5 Wocheu die Heilung vollendet, und spätestens nach 14 Tagen jeder meiner Kranken imstande gewesen, das Bett zu verlassen.

Ich glaube, dass jeder Arzt, der auf diesem Gebiete einige Erfahrung besitzt, mir zugeben wird, dass eine auch nur annähernd abgekürzte Heilungsdauer mit den bislang üblichen Methoden nicht zu erreichen ist.

Aber noch einen anderen, höchst beachtenswerthen Vortheil gewährt das vorstehend beschriebene Verfahren. Wie aus der Durchsicht der diesem Aufsat $z$ beigefügten Krankengeschichten hervorgelıt, ist es in jenem Falle gelungen, eine vollkommene oder wenigstens theilweise prima intentio herbeizuführen, a usnahmslos aber ist eine primäre Vereinigung der Muskulatur und des Darmrohrs erzielt, sodass kein einziger meiner Kranken je Mühe gehabt hat, den andrängenden Stuhl zurückzuhalten; alle besitzen vollständige Continenz auch für flüssigen Stuhl und Flatus.

Es würde noch die Frage zu erledigen sein, ob Fisteln und Abscesse jedweder Provenienz sich für diese Behandlungsart eignen.

Dass dieselbe fïr das Anfangsstadium der acuten septischen Phlegmonen des perirectalen Zellgewebes, die gelegentlich auch in den Mastdarm durchbrechen, nicht passt, ist selbstverständlich. Zweifelhaft könnte man nur sein bei aussergewöhnlich ausgedehnten tuberculösen Processen. Hier entscheidet die Rücksichtnahme auf das Allgemeinbefinden und den Zustand der übrigen Organe des Körpers. Dass man Tuberculöse in vorgeschrittenem Krankheitsstadium nicht ohne zwingenden Grund einer eingreifenden, radicalen Behandlungsweise unterwirft, ist allgemein anerkannter Grundsatz der Chirurgie. Im Anfang der Tuberculose aber ist die von mir empfohlene Operationsmethode der Fistula recti eben wegen der abgekïrzten Heilungsdauer die gewiesene Therapie.

Indem ich die mit manchem Misserfolg verbundenen operativen Vorversuche übergehe, gebe ich in kurzem Auszuge die Krankengeschichten der letzten hintereinander in der beschriebenen Weise behandelten Fälle aus den Jahren 1888 und 1889.

1. Frau Belling, 34 Jahre. Fistula recti incompleta, nach acuter phlegmonöser Entzündung; aussere Oeffnung $3 \mathrm{~cm}$ rechts yon der Allalöffnung; periproctitischer Abscess.

Operation 29. Februar 1888. Spaltung und Säuberung der Fistel und Abscesswände, theilweise Naht, Drainage des Abscesses.

Verla uf ungestört, glatte prima intentio, soweit genäht. Am 15. IÏ̈z 1888 alles verlleilt. Volle Continenz.

2. Frau Jung, 25 Jahre. Fistula recti completa; äussere Oeffnung $3 \mathrm{~cm}$ rechts von der Analöffnung, innere dicht über dem M. sphincter externus. Grosser periproctitischer Abscess. Gravidit. mens. V.

Operation 9. Juli 1888. Spaltung, Anfrischung und Naht der Fistel, breite Spaltung, Ausschabung und Drainage des Abscesses mit theilweiser Naht.

Verla uf ungestört. Die Fistel heilt prima intentione, auch der Abscess ist 14 Tage nach der Operation, am 23. Juli 1888, vollkommen ausgeheilt. Volle Continenz.

3. Herr B., 67 Jahre. Fistula recti completa; äussere Oeffnung $4 \mathrm{~cm}$ links von der Analöffnung, innere dicht über dem Sphincter externus. Ein Recessus erstreckt sich ungefähr $4 \mathrm{~cm}$ neben der Rectalwand hinauf.

Operation 6. October 1888. Excision. und Naht der Fistel und des Recessus.

Verlauf. Am 12. October ist die äussere Hautwunde prima intentione verheilt, in der Tiefe alles primär angelegt, nur die Ränder der Schleimhaut sind etwas auseinander gewichen. Am 26. October 1888 ist alles verheilt. Volle Continenz.

4. Arbeiter Wiegand, 53 Jahre. Fistula recti completa; äussere Oeffnung ungefähr $5 \mathrm{~cm}$ rechts von der Analöffnung, innere dicht über dem Sphincter externus. Periproctitischer Abscess, der mit feiner Oeffnung in die Fistel mündet. 
Operation 31. October 1888. Excision und Naht der Fistel, Spaltung des Abscesses, Anfrischung und Naht der Wände.

Verlauf. Schmerz- und reactionslos bei gutem Allgemeinbefinden; 6. November erster Verbandwechsel; prima intentio. 9. November 1888 geheilt entlassen mit voller Continenz.

5. Maurerlehrling Korn, 18 Jahre. Fistula recti completa; äussere Oeffnung $3 \mathrm{~cm}$ links von der Analöfnung, innere dicht über dem Sphincter externus.

Operation 4. April 1889. Excision der Fistel und Naht, 10. April prima intentione geheilt, Herausnahme der Nähte. Volle Continenz.

6. Frau K., 25 Jahre. Fistula recti completa; äussere Oeffnung $4 \mathrm{~cm}$ rechts von der Analöfnung, innere über dem Sphincter externus.

0 peration 26. Juli 1889. Excision der ganzen Fistel und Etagen11aht. 31. Juli 1889 Herausnahme der Nähte, glatte prima intentio. Am 2. August erster Stuhl, volle Continenz.

7. Frau L uthe, 33 Jahre. Fistula recti completa; äussere Oeffnung $\grave{\jmath} \mathrm{cm}$ links von der Analöffnung nach vorn gelegen; innere dicht über dem Sphincter externus an der vorderen Rectalwand.

Operation 1. August 1889, Spaltung der Fistel; ein buchtiger, von schwammigen Granulationen erfüllter Gang erstreckt sich neben dem Rectum $5 \mathrm{~cm}$ weit in die Höhe. Excision der Fistel und des Ganges, wobei auch die Rectalschleimhaut $5 \mathrm{~cm}$ breit durchschnitten werden muss. Etagennaht und Naht der Schleimhaut mit Catgut, Hautnaht mit Seide.

6. August. Glatte prima intentio mit voller Continenz.

8. Fräulein H., 55 Jahre. Fistula recti completa; äussere Oeffnung $5 \mathrm{~cm}$ links von der Analöffnung, innere $4 \mathrm{~cm}$ über dem M. sphincter externus. Ein grosser, hoch hinaufreichender periproctitischer Abscess umgreift an der hinteren Seite das Rectum und erstreckt sich nach rechts und links weit zwischen die Glutāalmuskulatur hinein.

Operation 11. September 1889. Spaltung und Excision der Fistel, Spaltung und Evidement des Abscesses, Anfrischung der Abscesswände, wobei viele Bindegewebssepta excidirt werden müssen. Naht der Fistel. Die grosse, nun glattwandige Abscesshöhle kann nur zum Theil durch die Naht geschlossen werden, weshalb auf beiden Seiten dicht über dem Tuber ischii je ein Drain vom tiefsten Punkte nach aussen geleitet wird.

Verlauf tadellos bei gutem Allgemeinbefinden. Nie Schmerz, nie Fieber. Am 16. September findet sich der Abscess zum grössten Theil primär angelegt, so dass das Drain rechts fortgelassen werden lann. Am 21. September bei Herausnahme der Nähte wird prima intentio, soweit hatte genäht werden kōnnen, constatirt. Auch der links vom Rectum gelegene Theil der Abscesshöhle ist soweit verheilt, dass das zweite Drain entfernt werden kann.

Am 16. October ist alles verheilt. Trotz der ausgiebigen Durchschneidung der Schliessmuskulatur ist nicht die geringste Incontinenz aufgetreten.

9. Arbeiter Krüger, 50 Jahre. Fistula recti completa, äussere Oeft nung $4 \mathrm{~cm}$ rechts von der Analöffnung, innere dicht über dem Sphincter externus. Periproctitischer Abscess.

Operation 18. September 1889: Excision und Naht der Fistel; Spal. tung, Anfrischung und Etagennaht des Abscesses.

Verlauf reactionslos, doch zeigt sich bei Herausnahme der Nähte arr. 25. September, dass nur theilweise prima intentio des Abscesses eingetreten ist. Am 17. October 1889 ist alles verheilt mit voller Continenz, so das. auch hier in 29 Tagen das Verfahren beendigt ist.

10. Herr D., 48 Jahre. Fistula recti completa; äussere Oeffnung $6 \mathrm{cn}$ links von der Analöffnung, innere ungefăhr $4 \mathrm{~cm}$ über derselben. Grosse buchtiger periproctitischer Abscess.

Operation am 14. October 1889: Nach Excision der ganzen Fistel wird der Abscess breit gespalten und gesäubert, wobei viele Bindegewebsbalken und Scheidewände exstirpirt, und livide, unterminirte Hautränder in weiter Ausdehnung abgetragen werden müssen. Hierauf sorgfältiger Schluss der Fistel und des Darmrohrs durch Catgutnaht. Trotz Zuhülfenahme ausgiebiger Hülfsschnitte gelingt es doch nur theilweise, den Abscess durch Etagennähte zu schliessen und die äussere Haut zu vereinigen; es bleibt eine flache, von aussen bequem zugängliche Höhlung zurück, die mit Jodoformgaze ausgefüllt wird.

Verlauf tadellos. Nie Schmerz oder Fieber bei ungestörtem Allgemeinbefinden. Am 20. October erster Verbandwechsel: soweit genäht, ist prima intentio eingetreten. Die Abscesshöhle granulirt gut. Patient verlässt das Bett. Am 21. October erster Stuhlgang, es ist bereits vollkommene Continenz vorhanden. - Am 24. October ist das Darmrohr ganz fest vernarbt, der Sphincter contrahirt sich vorzüglich. Die Abscesshöhle links ist sehr abgeflacht und verkleinert, mit guten Granulationen ausgekleidet. Am 9. November 1889 ist alles verheilt.

11. Herr Sch., 61 Jahre. Fistula recti completa; äussere Oeffnung $5 \mathrm{~cm}$ rechts von der Analöffnung, innere dicht über dem Sphincter externus.

Operation 9. November 1889: Excision der Fistel und Naht.

14. November prima intentio, Nāhte entfernt; erster Stuhl, wobei sich volle Continenz erweist.

Wie aus den Krankengeschichten erhellt, betrug die längste Heilungsdauer 35, die kürzeste 5, der Durchschnitt 15,7 Tage. Das Allgemeinbefinden ist nie gestort gewesen, nie ist Fieber, nie Schmerz aufgetreten, in keinem Falle ist Incontinenz zurückgeblieben.

Da ich glaube, dass diese Resultate als sehr befriedigende zu bezeichnen sind, gestatte ich mir, sie der Beurtheilung der Fachgenossen hiermit zu unterbreiten. 